J3eA, Journal sur l'enseignement des sciences et technologies de l'information et des systèmes, Volume 4, Hors-Série 1, 3 (2005)

DOI : http://dx.doi.org/10.1051/bib-j3ea:2005603

(C) EDP Sciences, 2005

Électronique de puissance pour la qualité de l'énergie des réseaux de bord d'avion

P. Ladoux ${ }^{1}$, F. Richardeau ${ }^{1}$ et L. Raulin ${ }^{2}$

${ }^{1}$ Laboratoire d'Électrotechnique et d'Électronique Industrielle

Unité Mixte de Recherche INPT-ENSEEIHT / CNRS

BP 7122

2 rue Camichel

F-31071 Toulouse CEDEX 7, France

${ }^{2}$ AIRBUS France

316 route de Bayonne

F-31060 Toulouse CEDEX 03, France 


\title{
Electronique de Puissance pour la qualité de l'énergie des réseaux de bord d'avion
}

\author{
Ph. Ladoux*, F. Richardeau*, L. Raulin** \\ *Laboratoire d'Electrotechnique et d'Electronique Industrielle \\ Unité Mixte de Recherche INPT-ENSEEIHT / CNRS \\ BP 7122 - 2 rue Camichel - 31071 TOULOUSE Cedex 7 - France \\ ** AIRBUS France \\ 316 Route de Bayonne, 31060 TOULOUSE CEDEX 03.
}

\section{PROBLEMATIQUE DE LA QUALITE RESEAU}

\section{I a) Introduction}

La problématique de qualité réseau est un sujet qui est examiné avec le maximum d'attention par AIRBUS, à chacune des étapes de conception d'une nouvelle famille d'avion. Les précautions de design qui en résultent sont de nos jours d'autant plus importantes en raison de l'augmentation de la puissance électrique installée, de l'introduction de nouveaux types de charges, ainsi que de la complexification du système électrique et de son architecture.

\section{I b) Le système électrique avion}

Basiquement, le système électrique d'un avion peut être segmenté en trois parties: la génération (générateurs électriques principaux, auxiliaires et de secours), la distribution (feeders et autres lignes d'alimentation, contacteurs de reconfiguration dynamique du schéma de puissance, dispositifs de protection contre les court-circuits) et les consommateurs de puissance (charges techniques et commerciales).

Les avions commerciaux actuels sont équipés d'un réseau alternatif dont la tension simple est de 115 V. Pour la majorité d'entre eux, ce réseau électrique est à fréquence fixe: $400 \mathrm{~Hz}$. Toutefois, la rationalisation des étages d'entraînement des générateurs principaux apportée sur les dernières générations d'AIRBUS conduit à un réseau à fréquence variable, dont le rapport des fréquences est proche de deux, typiquement de l'ordre de 400 à $800 \mathrm{~Hz}$.

En matière de charges, les derniers choix technologiques en matière de réseau, l'augmentation de la demande de puissance au niveau des utilisateurs, ainsi que la fiabilisation des dispositifs mettant en œuvre de l'électronique de puissance conduisent de nos jours à des configurations pour lesquelles le taux de charges non-linéaires est important : typiquement 40 à $70 \%$ dans certains cas de reconfiguration du réseau. Ceci provient notamment de la généralisation des convertisseurs de pilotage des régimes de fonctionnement des moteurs électriques mis en œuvre dans les dispositifs de contrôle de surface, de ventilation ou dans les pompes: pompes hydrauliques, pompes carburant... De manière plus détaillée, les principaux consommateurs-pollueurs peuvent, à ce jour, être classés comme suit :

Charges triphasées: système de conditionnement d'air (ventilateurs de recirculation de l'air cabine), actionneurs électriques de contrôle de surfaces, convertisseurs AC/DC, ventilateur de refroidissement centralisé des équipements, charges commerciales «galley» (production de froid, cuisson)...

Charges monophasées : systèmes d'information et de divertissement des passagers, éclairages cabine..

Si la gamme de puissance des charges s'étend aujourd'hui de quelques VA jusqu'à 10 à $20 \mathrm{kVA}$, il est à noter que cette tendance - augmentation de la puissance des charges, caractère non-linéaire des principaux consommateurs - s'accentuera encore dans le cadre de l'avion plus électrique avec l'introduction de nouvelles fonctions électriques : démarrage des réacteurs, dégivrage de la voilure, rentrée de train, etc...

\section{Ic) Origine du besoin de maîtrise de la qualité réseau}

La maîtrise de la qualité réseau nécessite de limiter le niveau de courant harmonique que les charges avion sont susceptibles de réjecter de façon à garantir que le taux global de distorsion de la tension réseau délivrée reste acceptable.

Les précautions de design qui sont prises permettent ainsi de s'affranchir des conséquences auxquelles est exposé un système pour lequel le niveau de qualité réseau serait insuffisant. En effet, 
dans un tel cas, la diminution du facteur de puissance induit une augmentation globale des pertes. L'augmentation du stress électrique et thermique des composants conduit à une diminution de la fiabilité des équipements. D'autre part, la maîtrise de la qualité réseau permet d'éviter une dégradation potentielle des performances électromagnétiques d'actionneurs ou de moteurs vis-à-vis de leurs objectifs de design, ou encore des décisions erronées de systèmes de contrôle commande. En effet, les changements qui peuvent intervenir sur l'enveloppe d'une forme d'onde en cas de distorsion réseau anormalement élevée peuvent perturber le bon fonctionnement de circuits de mesure (mesure de valeur efficace vraie, de valeur crête...) ou d'algorithmes basés sur une détection de passage par zéro d'une forme d'onde.

\section{I d) Exigences relatives à la maîtrise de la qualité réseau}

En matière de maîtrise de la qualité réseau, les principales exigences sont formulées au niveau du comportement électrique des utilisateurs, même si certaines précautions complémentaires sont prises au niveau des générateurs ainsi que du dimensionnement des lignes de puissance afin de limiter leur caractère impédant.

La maîtrise de la qualité réseau comporte deux aspects : la définition de caractéristiques précises du réseau avion en terme de pollution maximale admissible d'une part, les critères de design correspondant aux exigences auxquelles les équipements doivent répondre d'autre part.

Pour ce qui est du premier point, les caractéristiques réseau définies par AIRBUS sur un avion type A380 correspondent, en régime établi et dans le pire cas, à un taux global de distorsion de la tension délivrée de $10 \%$ en fonctionnement normal et de $15 \%$ en condition secours. Pour atteindre cet objectif, les contraintes imposées aux équipements visent à limiter leur niveau de courant harmonique réjecté. Les gabarits maximums qui doivent ainsi être respectés par chaque abonné du réseau électrique sont en droite ligne avec les exigences ISO 1540 / DO 160 et conduisent les équipementiers à des choix spécifiques au niveau des étages d'entrée des équipements non-linéaires. Ainsi, sauf exception, les étages d'entrée constitués de redresseurs basiques monophasés ou 6-Pulses ne sont pas autorisés en raison du fort niveau de pollution qu'ils engendrent, notamment sur les harmoniques de rang faible: harmoniques 3 (équipements monophasés uniquement), 5 et 7 entre autres. La figure 1 illustre les formes d'ondes qui y correspondent.

De ce fait, les contraintes de conception introduites conduisent à la mise en œuvre systématique d'étages actifs à absorption quasi-sinusoïdale pour les équipements monophasés. Pour les charges triphasées non-linéaires, les exigences de réjection harmonique correspondent au moins à l'utilisation d'étages de redressement 12-Pulses permettant de l'annulation de certains harmoniques de faible rang.

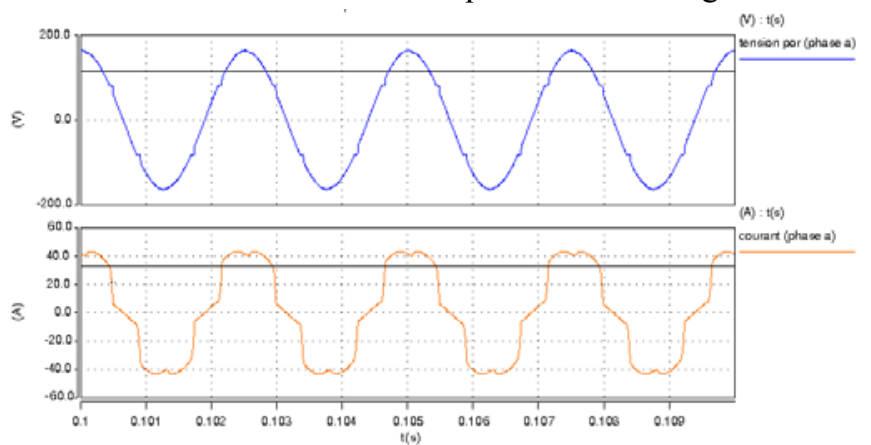

Figure 1.1 : Formes d'onde tension / courant d'un redresseur à diodes 6-Pulses en utilisation "redresseur de courant"

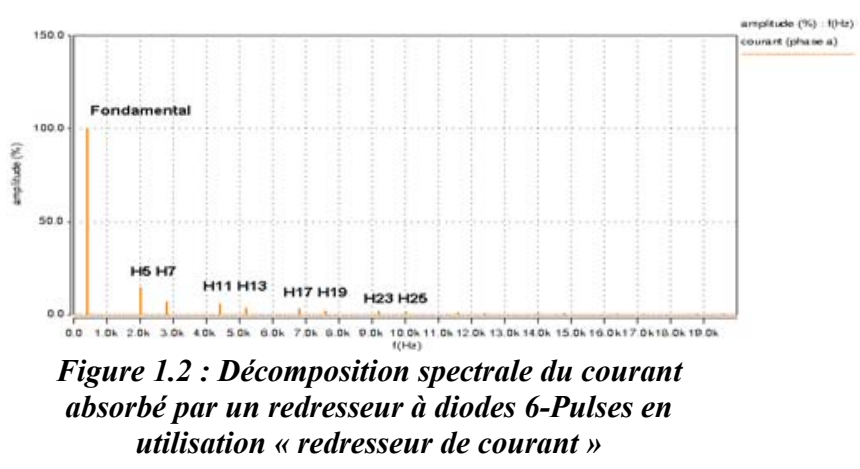

I e) Outils \& méthodes

Dans ce contexte, un certain nombre d'outils et de méthodes est mis en œuvre par AIRBUS, afin de participer à l'élaboration et à la validation d'exigences de qualité réseau ou encore pour évaluer l'efficacité et la pertinence de technologies nouvelles. Ainsi, le développement de maquettes virtuelles permet depuis plusieurs années déjà de travailler en avance de phase à la fois au niveau des études amont ou encore lors des choix effectués en début d'un programme avion. Pour ce faire, le logiciel de simulation mixte SABER est largement mis en oeuvre afin de conduire l'intégration de modèles de génération, de distribution et d'utilisateur de puissance en vue des études de qualité réseau. L'intérêt de cette démarche est de permettre une prédiction et une analyse détaillée du niveau des perturbations harmoniques auquel on peut s'attendre sur le réseau avion, très tôt dans le planning de conception de ce dernier, c'est à dire avant même le commencement de l'intégration du réseau électrique et de l'installation des équipements. Tout excès de pollution harmonique peut alors donner lieu à des actions correctives appropriées, soit au niveau du système, soit au niveau d'une famille précise d'équipements et ce, avec un niveau d'anticipation maximum. 

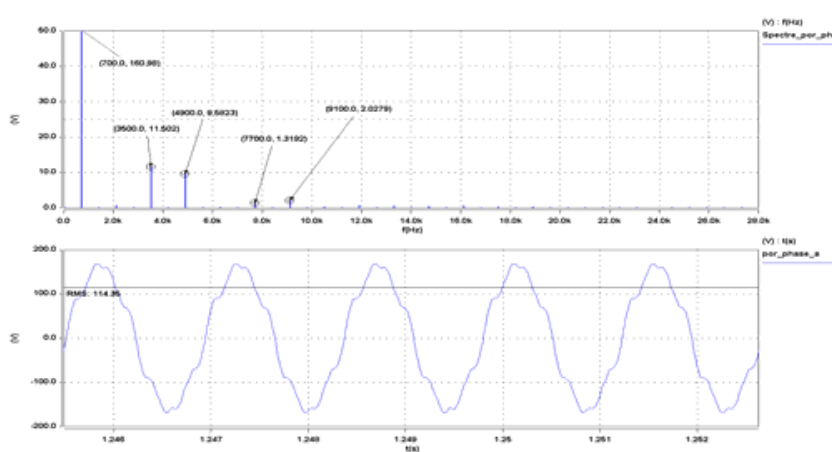

Figure 1.3 : Résultats de simulation SABER : forme d'onde temporelle et décomposition spectrale de la tension réseau d'un avion type $\mathbf{A 3 8 0 .}$

D'autre part, les tests réalisés sur les bancs d'essais constituent le complément à ces activités de simulation de manière à permettre le recalage adéquat des modèles et la validation des résultats de simulation sur certains scénarios type. Les mesures de réjection sur l'ensemble de la plage de fréquence, ainsi que les tests de susceptibilité qui sont réalisés permettent donc de contribuer efficacement à la validation globale des exigences de qualité réseau. L'utilisation conjointe de la simulation et de tests sur bancs d'essais permet en outre de mener finement les études en fonction de la phase de vol et pour différentes configurations avion, ainsi que d'optimiser la corrélation avec les enregistrements réalisés sur avions.

\section{FILTRAGE ACTIF DES HARMONIQUES}

\section{II a) Introduction}

Comme c'est déjà le cas pour les réseaux industriels de fréquence $50 \mathrm{~Hz}$, il peut être intéressant pour les réseaux de bord d'avion d'envisager une compensation active des harmoniques. Cela évite toute modification sur les étages d'entrée des récepteurs non linéaires et il est donc possible de continuer à fonctionner avec des équipements existant. Sur le principe, le filtre actif sera réalisé autour d'un onduleur de tension MLI [1]. Le fonctionnement de l'onduleur MLI pour une telle application est très particulier, dans la mesure où il fonctionne à puissance active nulle, et n'échange donc que de la puissance fluctuante avec son environnement. Le schéma ne fait apparaître pour la source du bus continu qu'un simple condensateur dont il faut contrôler la tension pour qu'elle reste dans une fourchette déterminée. Pour le filtrage actif, il faut pouvoir «créer» des courants présentant des sauts (donc des di/dt élevés), ce qui nécessite des performances en terme de bande passante très supérieures à celles exigées par un fonctionnement plus classique de l'onduleur MLI. Comme nous allons le voir, cela se répercute notamment sur le niveau de tension du bus continu et sur le choix de semi-conducteurs permettant une fréquence de commutation élevée.
II b) raccordement d'un onduleur de tension sur un réseau alternatif

Nous considérons ici un onduleur monophasé à point milieu (Fig. 2.1). Du point de vue des bornes de sortie, l'onduleur de tension se comporte comme une source de tension bidirectionnelle en tension et réversible en courant. Le raccordement de l'onduleur de tension sur un réseau alternatif supposé infiniment puissant, (impédance interne nulle) doit donc se faire par l'intermédiaire d'une inductance qui se comporte comme un filtre passe bas et limite l'ondulation de courant à la fréquence de commutation. Si la fréquence de commutation est très grande devant la fréquence du réseau, l'ondulation de courant maximale est donnée par la relation suivante :

$$
\Delta I_{\max }=\frac{E}{4 f_{d} L}
$$

Pour contrôler la puissance active échangée avec le réseau, il faut que l'onde fondamentale de tension délivrée par l'onduleur ait une fréquence égale à la fréquence du réseau. L'amplitude du courant peut ensuite être réglée par l'intermédiaire d'une boucle de régulation incluant par exemple un correcteur Proportionnel Intégral.

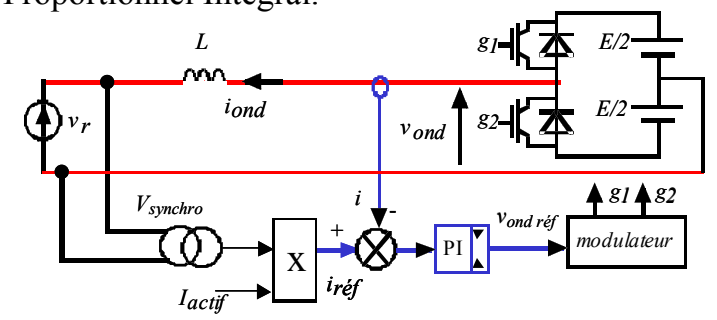

Figure 2.1 : principe de raccordement d'un onduleur de tension sur un réseau alternatif.

La consigne de courant est donc élaborée à partir de la mesure de la tension délivrée par le réseau et elle permet de régler le niveau et le signe de la puissance active.

\section{II c) Principe du filtre actif parallèle}

Un filtre actif parallèle est équivalent à une source de courant commandée qui permet d'injecter sur le réseau la somme des harmoniques de rang bas générés par la charge non linéaire (Fig. 2.2).

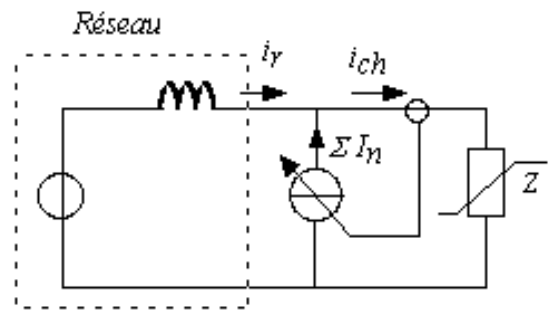

Figure 2.2 : Principe du filtrage actif parallèle. 
Dans ces conditions, le courant vu par le réseau correspond à la composante fondamentale du courant de la charge non linéaire. La réalisation d'un filtre actif parallèle à partir d'un onduleur de tension s'effectue en rajoutant une entrée supplémentaire au niveau de la boucle de courant (Fig. 2.3). En effet, vis à vis du réseau, l'onduleur de tension muni de sa boucle de courant se comporte comme une source de courant commandée.

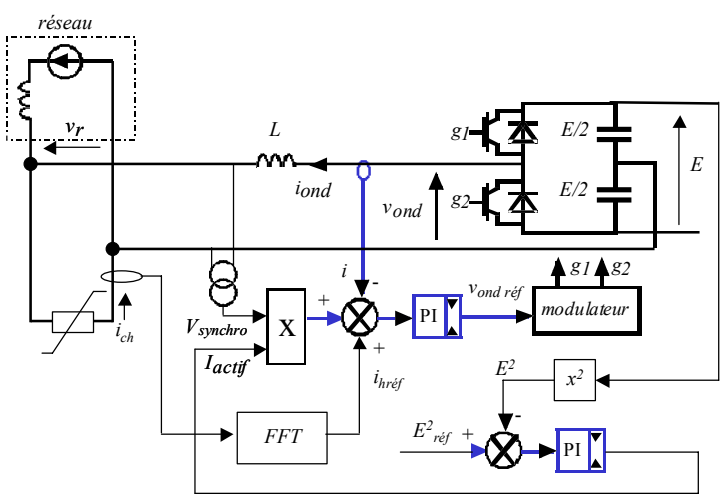

Figure 2.3: Principe de réalisation d'un filtre actif parallèle à l'aide d'un onduleur de tension MLI (schéma monophasé).

L'élaboration de la consigne en courants harmoniques $\left(i_{\text {hréf }}\right)$ peut être réalisée à partir d'une FFT sur la mesure du courant dans la charge. Avec cette méthode, il est donc possible d'effectuer un filtrage sélectif en amplitude et en phase au niveau de chaque rang d'harmonique.

Pour obtenir un bon fonctionnement du filtre actif, il est indispensable de contrôler la tension du bus continu. Une boucle de régulation de $\mathrm{E}^{2}$ assure en régime permanent l'égalité des puissances côté alternatif et côté continu (compensation des pertes au niveau de l'onduleur). Lors des régimes transitoires (variations de charge), la tension du bus continu est ainsi maintenue dans une fourchette acceptable pour les semi-conducteurs et les condensateurs.

\section{II-d) Exemple de dimensionnement pour un réseau} de bord d'avion type A380.

Le réseau triphasé de l'A380 (115V/200V) a la particularité de fonctionner à fréquence variable. Pour dimensionner notre filtre actif, nous nous plaçons dans le cas où la fréquence du réseau est maximale ( $\mathrm{f}=800 \mathrm{~Hz}$ ). Au point de raccordement de la charge non linéaire, le réseau possède une impédance interne telle que $r_{i}=30 \mathrm{~m} \Omega$ et $l_{i}=$ $20 \mu \mathrm{H}$. Nous considérons le cas très défavorable d'une charge non linéaire triphasée d'une puissance de $7,4 \mathrm{~kW}$ et absorbant des courants non sinusoïdaux dont le taux de distorsion est de $67 \%$.
La décomposition spectrale du courant de ligne de (phase 1par exemple) est la suivante :

$$
\begin{aligned}
& i \operatorname{ch}(t)=I 1 \sqrt{2} \cos \left(\omega t-\frac{\pi}{3}\right)+I 3 \sqrt{2} \sin 3 \omega t \\
& +\sqrt{2} \sum_{h=1}^{4} I_{6 h \pm I} \sin (6 h \pm 1) \omega t
\end{aligned}
$$

La charge absorbe des harmoniques de courant jusqu'au rang 25 , soit sur une plage de fréquence allant jusqu'à $20 \mathrm{kHz}$. Le courant à 3.f, typiquement généré par des charges non linéaires monophasées, correspond à une composante homopolaire qui circule dans le conducteur neutre $\mathrm{du}$ réseau. Le point milieu du bus continu du filtre actif devra donc obligatoirement être relié au neutre du réseau afin de compenser ce courant.

Pour la charge considérée, l'amplitude de chaque harmonique est donnée dans le tableau suivant :

\begin{tabular}{|l|l|}
\hline Rang & Valeur efficace (A) \\
\hline 1 & 25 \\
\hline 3 & 9 \\
\hline 5 & 9 \\
\hline 7 & 7,5 \\
\hline 11 & 5 \\
\hline 13 & 5 \\
\hline 17 & 2,5 \\
\hline 19 & 2,5 \\
\hline 23 & 2 \\
\hline 25 & 2 \\
\hline
\end{tabular}

Tableau I: Valeurs efficaces des harmoniques absorbés par la charge.

La figure 2.4 présente des résultats de simulations obtenus avec Psim: sans filtrage actif, la charge perturbe très fortement la tension réseau dont le taux de distorsion atteint alors $20 \%$.

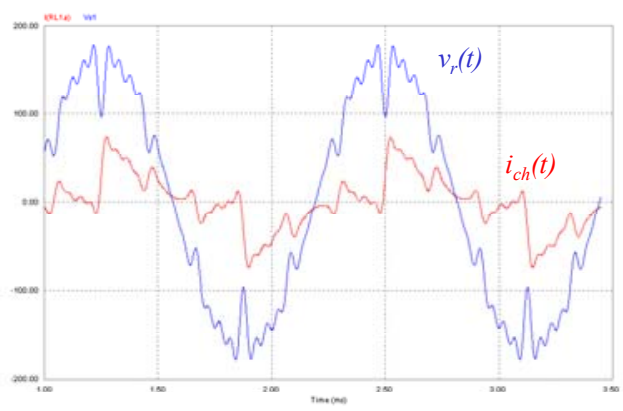

Figure 2.4 : conséquence de la charge non linéaire sur le réseau de bord.

Pour que la tension $\mathrm{V}_{\mathrm{r}}(\mathrm{t})$ soit sinusoïdale, le filtre actif doit compenser les harmoniques de courant jusqu'au rang 25 , le courant fourni par l'onduleur s'exprime par la relation (3) : 


$$
\begin{aligned}
& \text { iond }(t)=I 3 \sqrt{2} \sin 3 \omega . t \\
& +\sqrt{2} \sum_{h=1}^{4} I_{6 h \pm 1} \sin (6 h \pm 1) \omega . t
\end{aligned}
$$

La composante «basse fréquence » de la tension délivrée par l'onduleur est calculée à partir de la relation (4) :

$$
v_{\text {ond }}=v_{r}+L \frac{d i_{\text {ond }}}{d t}
$$

Soit :

$$
\begin{aligned}
& v_{n d}(t)=V r \sqrt{2} \cos \omega \cdot t+L \cdot \omega \cdot I 3 \sqrt{2} \cos 3 \omega . t \\
& +L . \omega \cdot \sqrt{2} \sum_{h=1}^{4} I_{6 h \pm 1} \cos (6 h \pm 1) \omega . t
\end{aligned}
$$

Il apparaît alors que $v_{\text {ond }}$ est une somme directe de fonctions sinusoïdales. Plus les rangs à compenser sont nombreux, plus $v_{\text {ond }}$ sera d'amplitude élevée.

La bande passante du filtre actif dépend bien évidemment de la bande passante des boucles de courant mais aussi de la tension E du bus continu. Plus les harmoniques à compenser sont de rang élevé, plus il faut une tension $\mathrm{E}$ élevée. La figure 2.5 présente la forme d'onde théorique de $v_{\text {ond }}(t)$ calculée à partir de la relation (5) et pour une inductance $\mathrm{L}=55 \mu \mathrm{H}$.

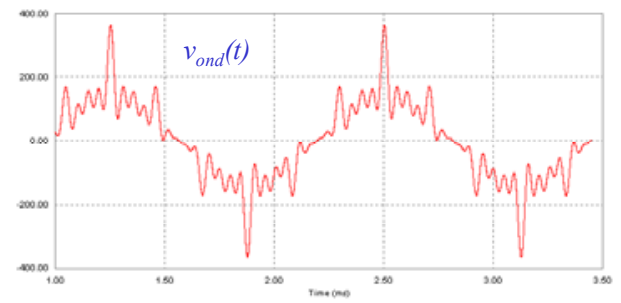

Figure 2.5: Allure théorique du signal modulant de l'onduleur MLI

La tension du bus continu est calculée à partir de la relation (6) où $\mathrm{m}_{\mathrm{amax}}$ est la profondeur de modulation maximale au niveau de la commande de l'onduleur :

$$
E=\frac{2 \cdot \operatorname{Max}\left(v_{\text {ond }}(t)\right)}{m_{a \max }}
$$

Sur la figure 2.5 $\operatorname{Max}\left(v_{\text {ond }}(t)\right)$ est de $360 \mathrm{~V}$. Ainsi, en considérant une profondeur de modulation $\mathrm{m}_{\mathrm{amax}}$ de 0,9 , la tension du bus continu $\mathrm{E}$ doit être fixée à $800 \mathrm{~V}$. De manière générale, le filtrage actif des harmoniques nécessite un onduleur de tension à large bande passante [2] et il est nécessaire de choisir une fréquence de commutation au moins 3 à 4 fois supérieure à celle du rang d'harmonique le plus élevé. Pour répondre à de telles contraintes, tension et fréquence de commutation élevées, une structure d'onduleur multiniveaux à cellules imbriquées [3] est la plus appropriée. Dans notre cas, cette structure peut être basée sur des bras d'onduleur à trois cellules imbriquées (Fig. 2.6) utilisant des CoolMos sous une tension de 800/3= $266 \mathrm{~V}$ et à une fréquence de commutation $\mathrm{f}_{\mathrm{d}}$ de 75 $\mathrm{kHz}$.

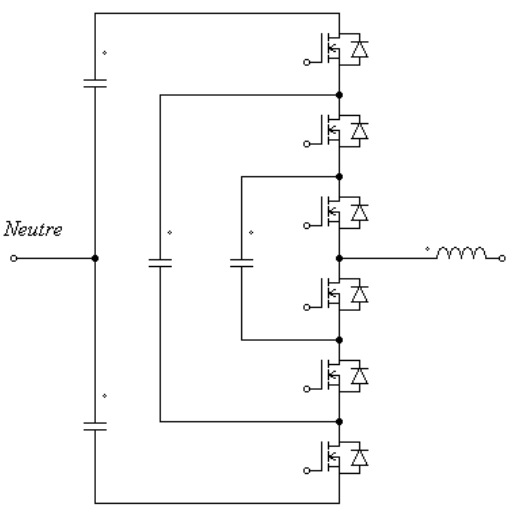

Figure 2.6 : Bras d'onduleur tri-cellulaire à MOS.

En sortie de l'onduleur, la fréquence apparente de découpage est alors de $225 \mathrm{kHz}(3 \times 75 \mathrm{kHz})$ et l'ondulation maximale de courant à $225 \mathrm{kHz}$ est de 5,4 A pour une inductance $\mathrm{L}$ de $55 \mu \mathrm{H}$ $\left(\Delta I \max =\frac{E}{36 . f d L}\right)$. La figure 2.7 présente des résultats de simulations obtenus avec le logiciel Psim (le correcteur de la boucle de courant est réglé pour obtenir une bande passante de $f_{d} / 2$ soit 37,5 $\mathrm{kHz}$ ). L'allure du courant fourni par le filtre est conforme au résultat de l'étude théorique. Toutefois, la tension $v_{r}(t)$ présente une large ondulation à la fréquence apparente de découpage. Dans notre cas, le diviseur de tension inductif inductance interne réseau / inductance de filtre, n'atténue pas suffisamment l'ondulation de tension haute fréquence $\left(\frac{l i}{L+l i}=0,26\right)$ et il est nécessaire d'ajouter un élément de filtrage passe bas en sortie $\mathrm{du}$ filtre actif. La fréquence de coupure de ce filtre doit être inférieure à $225 \mathrm{kHz}\left(3 . \mathrm{f}_{\mathrm{d}}\right)$ et supérieure à $20 \mathrm{kHz}(25$ x $800 \mathrm{~Hz})$.

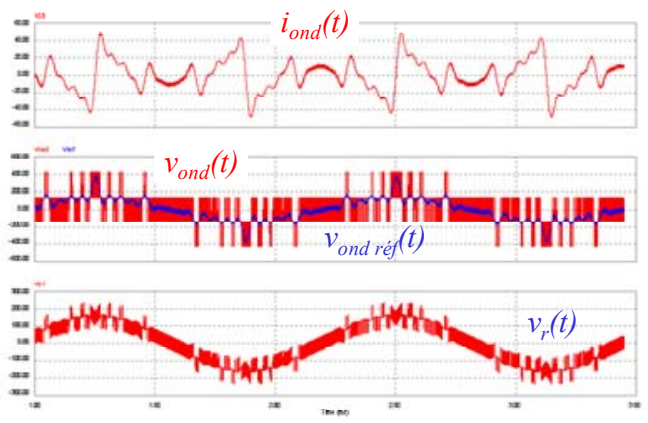

Figure 2.7 : Simulation Psim du filtre actif avec bras d'onduleur tri-cellulaire.

La figure 2.8 présente les résultats de simulation dans le cas d'un filtre LC en sortie de l'onduleur. 
L'inductance est toujours de $55 \mu \mathrm{H}$ et la capacité du condensateur est de $310 \mathrm{nF}$.

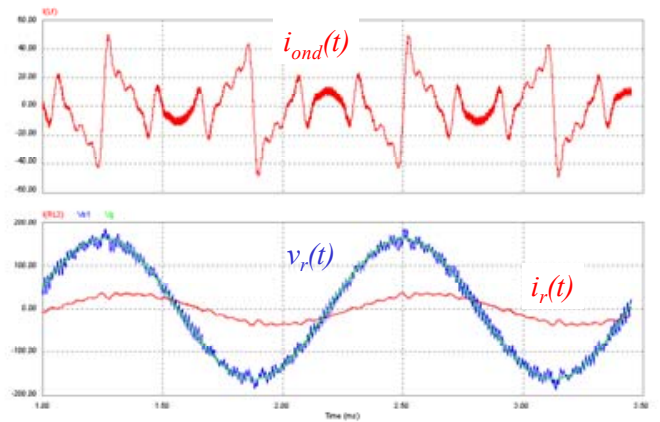

Figure 2.8: Simulation Psim du filtre actif avec bras d'onduleur tri-cellulaire et Filtre LC.

Le courant $i_{r}(t)$ et la tension $v_{r}(t)$ présentent après compensation un taux de distorsion respectivement de $12 \%$ et $10,7 \%$, ce qui n'est pas totalement satisfaisant. La décomposition spectrale de $i_{r}(t)$ est donnée dans le tableau ci-dessous :

\begin{tabular}{|l|l|}
\hline Rang & Valeur efficace (A) \\
\hline 1 & 25 \\
\hline 3 & $\sim 0$ \\
\hline 5 & $\sim 0$ \\
\hline 7 & $\sim 0$ \\
\hline 11 & 0,54 \\
\hline 13 & 0,58 \\
\hline 17 & 0,65 \\
\hline 19 & 0,87 \\
\hline 23 & 1,05 \\
\hline 25 & 1,2 \\
\hline
\end{tabular}

Tableau II : décomposition spectrale du courant de ligne après filtrage.

Il apparaît clairement que les harmoniques de rang élevé ne sont pas éliminés correctement. Ceci est principalement dû à réponse fréquentielle de la boucle de courant qui introduit pour ces rangs là un déphasage non négligeable entre la consigne de courant $i_{\text {hréf }}(t)$ et le courant $i_{\text {ond }}(t)$. Toutefois, la FFT du courant de charge permettant de connaître avec précision la phase de chaque harmonique, il est alors possible de compenser au niveau de chaque rang l'erreur de phase introduite par la boucle de courant et donc d'éliminer efficacement les harmoniques jusqu'au rang 25 .

\section{STRUCTURES PFC}

Plutôt que de dépolluer un réseau d'énergie de ses harmoniques par un dispositif actif "externe" aux équipements de bord existants, nous envisageons maintenant une autre alternative : la conception d'interfaces $\mathrm{AC} / \mathrm{DC}$ à prélèvement sinusoïdal du courant, "intégrées" au sein même d'une nouvelle génération d'équipements. Ces interfaces sont injustement nommées PFC (power factor controller, terme trop vague ...) et permettent de réaliser deux fonctions simultanément : l'absorption d'un courant quasi-sinusoïdal en phase avec la tension d'alimentation (respect des normes édictées au premier paragraphe) et la régulation d'une tension de bus locale ou distribuée pour alimenter d'autres convertisseurs (futurs onduleurs pour EHA/EBHA/EMA entres autres). La figure 3.1 illustre la fonction $\mathrm{PFC}$ à réaliser.

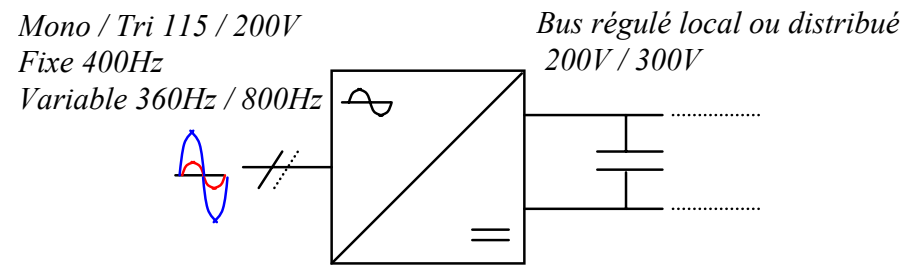

Figure 3.1 : Fonction PFC pour réseau de bord d'avion.

Dans ce contexte, l'objectif de ce paragraphe est de dresser un bref comparatif "qualitatif" de structures PFC sur les trois critères suivants : performances intrinsèques, dimensionnement des éléments de filtrage (en particulier les selfs !), et bien entendu la sûreté de fonctionnement. Sur ce dernier critère nous nous limiterons à examiner simplement la conséquence d'un défaut sur un transistor et à montrer l'aptitude de certaines topologies à préserver un haut niveau de sécurité, voire une poursuite du fonctionnement.

\section{III a) Structures PFC monophasées}

La structure de base bien connue [4] est rappelée en figure 3.2. Le réseau redressé est raccordé à un convertisseur élévateur boost. Une self supplémentaire à celle du réseau doit être placée en amont ou en aval du pont de redresseur ainsi qu'un filtre réseau (mode commun et différentiel). La modulation du rapport cyclique du seul transistor permet d'imposer en entrée en courant à enveloppe sinusoïdale et en sortie une tension régulée en valeur moyenne. Un contrôle MLI à fréquence de découpage fixe est conseillé afin d'éviter la présence d'inter-harmoniques basse fréquence sur le courant réseau permettant de faciliter le filtrage. Une haute fréquence de découpage est recherchée pour minimiser la masse de la self de lissage et obtenir une bande passante de commande élevée, bien que ce dernier point soit nettement moins 
critique que pour la fonction filtre actif exposée précédemment. En effet, la régulation du courant doit "seulement" suivre une référence à la fréquence $\mathrm{du}$ réseau, i.e. la fréquence du fondamental $400 \mathrm{~Hz}$ (avions actuels), ou $800 \mathrm{~Hz}$ (A380). Cette fréquence de découpage trouve évidemment une limite haute au niveau des pertes par découpage et donc de la surface d'échange du radiateur associé au transistor, et éventuellement au niveau du mode de refroidissement. Selon les puissances à traiter, des composants CoolMos 600V et IGBT $300 \mathrm{~V} / 600 \mathrm{~V}$ à $50 \mathrm{kHz}$ peuvent prétendre à des performances tout à fait honorables. Deux inconvénients caractérisent cette topologie de base. Le premier est évidemment lié au raccordement monophasé : le condensateur de bus est dimensionné pour la fréquence double du réseau, et non pas pour le découpage. Le second inconvénient est lié au passage systématique en butée du rapport cyclique à chaque fois que la tension du réseau passe par zéro.

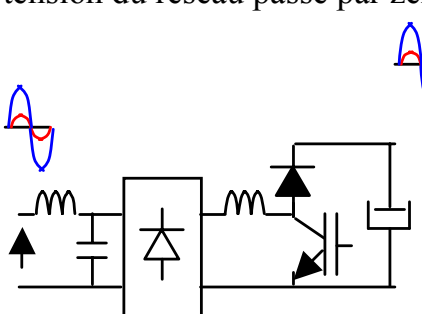

a)

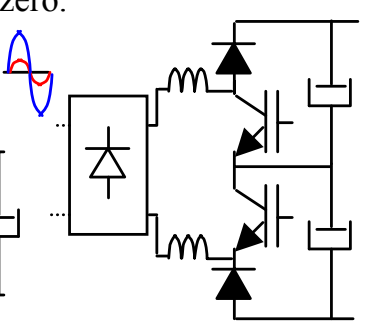

b)

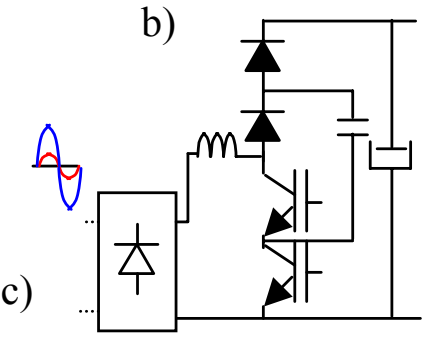

Figures 3.3 : a) PFC "simple" boost ; b) PFC "double" boost ; c) PFC multicellulaire.

Sur le plan de la sûreté de fonctionnement, un défaut de maintien à l'ouverture du transistor transforme la topologie en un simple redresseur non contrôlé, tandis que le maintient en fermeture du transistor amène naturellement la protection amont à disjoncter sans que le bus continu soit touché par le défaut. La conséquence d'un défaut sur le transistor n'est donc pas critique, et on aura intérêt à privilégier un mode de défaut de type "ouverture" pour le transistor par un choix technologique adapté de l'assemblage du transistor et du driver de commande.

Les variantes $3.3 \mathrm{~b}$ et $3.3 \mathrm{c}$ [5]-[6] visent à augmenter la fréquence de découpage apparente de façon à réduire la masse de la self et éventuellement à augmenter la bande passante mais ce n'est pas la priorité ici. Ces deux topologies mettent en œuvre une mise en série de deux cellules de commutation élémentaires de type boost. En décalant les ordre de commande, on passe d'une tension deux niveaux à une tension trois niveaux à fréquence double : la valeur de la self est ainsi divisée par quatre. Les transistors supportent la moitié de la tension de bus, de calibre plus faible, ils sont également plus rapides. A radiateur identique, il est donc possible de bénéficier d'une fréquence de découpage encore plus élevée.

\section{III b) Structures PFC triphasées}

L'extension au triphasé des topologies précédentes n'est pas envisageable simplement, sauf si un isolement galvanique est requis. Dans ce cas il suffit de coupler trois PFC monophasé entre phases par leurs sorties isolées. Dans le cas plus général, une topologie en pont est utilisée comme illustrée en figure 3.4.

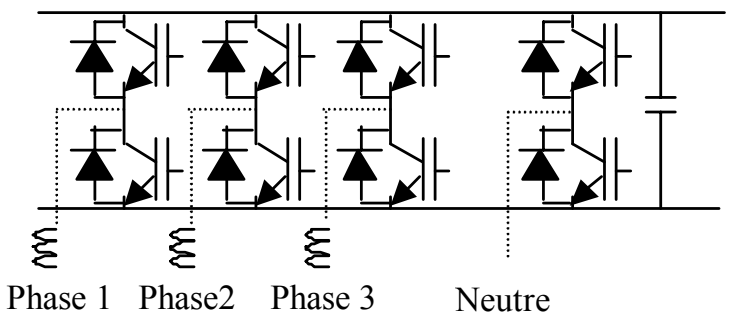

Figure 3.4 : PFC triphasé en simple pont à point milieu actif.

Cette topologie est bien connue et fait l'objet d'une littérature abondante [7]. Comparativement au PFC simple boost, elle permet de supprimer les deux inconvénients précités, bien entendu, cette topologie apporte une réversibilité permanente de la puissance. Cette propriété permettrait par exemple de supprimer la résistance de dissipation de l'onduleur EHA dans la configuration actuelle. Par contre, sur le plan de la sûreté de fonctionnement, la présence de deux transistors en série par bras de pont rend le système beaucoup moins sécurisé que les topologies précédentes. En effet, en cas de défaut de commande ou de claquage sur un des deux transistors, le bus onduleur se décharge violemment dans le bras avec une relative forte énergie : $1 \mathrm{mF}$ sous $300 \mathrm{~V}$ libère 45 joules, énergie suffisante pour provoquer une petite explosion de boîtier. Il est donc nécessaire d'adjoindre aux transistors des drivers avec détection rapide du défaut de court-circuit provoquant une disjonction adaptée. En supposant le défaut correctement géré au niveau d'un bras, la poursuite du fonctionnement sur les deux autres bras peut être obtenue en monophasé ou en diphasé moyennant une reconfiguration de la modulation et le raccordement du neutre sur un point milieu actif.

Dans le cas où la réversibilité permanente n'est pas requise ou si un haut niveau de sûreté est demandé, nous proposons en figure 3.5 une topologie à double boosts séquentiels. Chaque bras est 
constitué de deux boosts en série fonctionnant alternativement selon la polarité de tension [6]-[7]. Un simple redresseur deux diodes permet le raccordement de la phase au montage par rapport à un point milieu capacitif.

La figure 3.5 montre les formes d'ondes principales où l'on retrouve l'inconvénient du passage en butée et dans le cas présent un point milieu à une fréquence triple $(1400 \mathrm{~Hz}$ dans le pire des cas). En présence d'un défaut de maintien à l'ouverture des transistors (perte des alimentations auxiliaires par exemple, défaut de connectique) le montage passe naturellement en redresseur simple, à l'inverse si un transistor est maintenu conducteur ou physiquement défaillant c'est la disjonction de la phase qui jouera son rôle. Il faut noter que le raccordement du neutre au point milieu permet, comme précédemment, un fonctionnement en diphasé si un bras est défaillant.

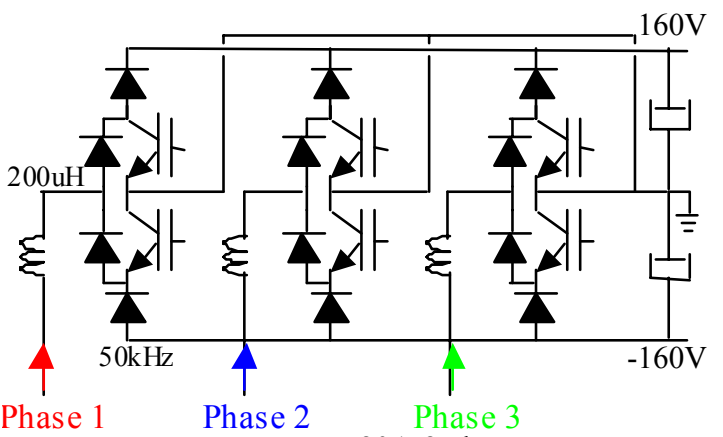

Phase $1 \quad$ Phase 2 Phase 3

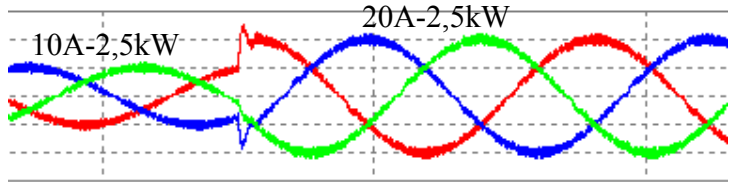

Courant dans le point milieu / courant filtré

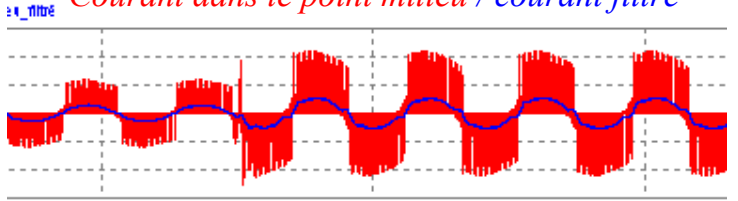

Ill__ntues Tension homopolaire / Tension filtrée
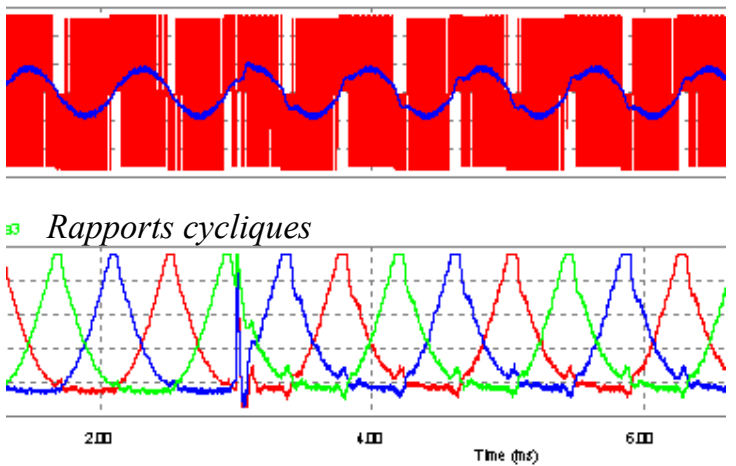

Figure 3.5 : PFC triphasé à boosts séquentiels (avec surmodulation).[10A/div]-400Hz.

\section{CONCLUSION}

Comme cela a été le cas au cours de ces dernières années, il est indéniable que les exigences de qualité de l'énergie au niveau des réseaux de bord en aéronautique vont continuer d'évoluer. Les évolutions de celles-ci seront en outre probablement significatives en raison des changements en cours au niveau de la philosophie d'utilisation des différentes énergies hydraulique, pneumatique et électrique à bord d'un avion, ainsi que des ruptures technologiques futures induites par une généralisation de l'électronique de puissance embarquée. Au travers de cet article, il apparaît clairement que les structures PFC peuvent apporter à moindre coût des solutions garantissant la qualité de la tension du réseau de bord tout en conservant une bonne disponibilité (modes dégradés possibles). Ce n'est que dans les cas où il sera impossible de modifier l'électronique d'un équipement embarqué (ex : volume minimum) que la fonction filtre actif sera à envisager. L'étude présentée dans le paragraphe II met en évidence les contraintes de réalisation d'un tel équipement: structure de convertisseur complexe et électronique de commande associée ultra performante en terme de temps de calcul.

\section{REFERENCES}

[1] "Utilisation de l'onduleur de tension MLI pour la correction du facteur de puissance", Philippe Ladoux, Michel Metz, Revue 3EI, mars 2002.

[2] "Evaluation et optimisation de la bande passante des convertisseurs statiques. Application aux nouvelles structures multicellulaires."

Aimé Martin,

Thèse de Doctorat de l'INPT 14 novembre 2003.

[3] "Multi-level choppers for high voltage applications",

Thierry Meynard, Henri Foch, EPE Journal, Vol 2, n¹, 1992.

[4] "Etude de convertisseurs AC/DC à absorption sinusoïdale",

Serge Delbosc,

Thèse de l'ENS Cachan, juin 1999.

[5] "PFC multicellulaire", Dominique Estrade, Mémoire de Thèse CNAM, INP Toulouse, 2002.

[6] "Contribution à l'étude des redresseurs à facteur de puissance quasi-unitaire fonctionnant en mode multiniveaux. Application au chargeurs de batteries", Patrick Bartholomeus, Thèse de l'Université des Sciences et Technologies de Lille, juin 1996.

[7] "Etude de l'immunité des redresseurs triphasés à contrôle scalaire MLI. Nouvelles stratégies de contrôle", Luis Marroyo, Thèse en co-tutelle INP Toulouse - Université de Pampelune, 1990. 